\title{
Three-Dimensional (3-D) Reconstructions of EISCAT IPS Velocity Data in the Declining Phase of Solar Cycle 23
}

\author{
M.M. Bisi • B.V. Jackson • A.R. Breen • G.D. Dorrian • \\ R.A. Fallows · J.M. Clover · P.P. Hick
}

Received: 29 November 2009 / Accepted: 17 June 2010 / Published online: 14 July 2010

(C) The Author(s) 2010. This article is published with open access at Springerlink.com

\begin{abstract}
The European Incoherent SCATter (EISCAT) radar has been used for remotesensing observations of interplanetary scintillation (IPS) for a quarter of a century. During the April/May 2007 observing campaign, a large number of observations of IPS using EISCAT took place to give a reasonable spatial and temporal coverage of solar wind ve-
\end{abstract}

Remote Sensing of the Inner Heliosphere

Guest Editors: M.M. Bisi, and A.R. Breen.

M.M. Bisi · B.V. Jackson · J.M. Clover · P.P. Hick

Center for Astrophysics and Space Sciences, University of California, San Diego, 9500 Gilman Drive \#0424, La Jolla, CA 92093-0424, USA

B.V. Jackson

e-mail: bvjackson@ucsd.edu

J.M. Clover

e-mail: jclover@ucsd.edu

P.P. Hick

e-mail: pphick@ucsd.edu

M.M. Bisi ( $\varangle)$ · A.R. Breen · R.A. Fallows

Institute of Mathematics and Physics, Aberystwyth University, Penglais Campus, Aberystwyth, Ceredigion SY23 3BZ, Wales UK

e-mail: Mario.Bisi@aber.ac.uk

\section{A.R. Breen \\ e-mail: azb@aber.ac.uk \\ R.A. Fallows \\ e-mail: raf@aber.ac.uk}

G.D. Dorrian

Astrophysical Research Centre, Queen's University of Belfast, University Road, Belfast, County Antrim BT7 1NN, Northern Ireland, UK

e-mail: g.dorrian@qub.ac.uk

P.P. Hick

San Diego Supercomputer Center, University of California, San Diego, 9500 Gilman Drive \#0505, La Jolla, CA 92093-0505, USA 
locity structure throughout this time during the declining phase of Solar Cycle 23. Many co-rotating and transient features were observed during this period. Using the University of California, San Diego three-dimensional (3-D) time-dependent computer assisted tomography (C.A.T.) solar-wind reconstruction analysis, we show the velocity structure of the inner heliosphere in three dimensions throughout the time interval of 20 April through 20 May 2007. We also compare to white-light remote-sensing observations of an interplanetary coronal mass ejection (ICME) seen by the STEREO Ahead spacecraft inner Heliospheric Imager on 16 May 2007, as well as to in-situ solar-wind measurements taken with nearEarth spacebourne instrumentation throughout this interval. The reconstructions show clear co-rotating regions during this period, and the time-series extraction at spacecraft locations compares well with measurements made by the STEREO, Wind, and ACE spacecraft. This is the first time such clear structures have been revealed using this 3-D technique with EISCAT IPS data as input.

Keywords Coronal mass ejections · Interplanetary · Radio scintillation · Solar wind, disturbances · Velocity fields, solar wind

\section{Introduction}

Interplanetary scintillation (IPS) observations of astronomical radio sources have been used to enable measurements of the solar-wind velocity for over 45 years. IPS is the amplitude fluctuation of radio waves from a distant compact radio source caused by density inhomogeneities in the solar wind which give rise to turbulent structure(s) moving outward in the solar wind. Here, we use IPS data from the European Incoherent SCATter (EISCAT) radar (Rishbeth and Williams, 1985; Wannberg et al., 2002) and EISCAT Svalbard Radar (ESR) (Wannberg et al., 1997) from 20 April 2007 to 20 May 2007. It should be noted that there were few-to-no observations during the period 28 April 2007 to 03 May 2007 inclusive.

The EISCAT UHF system consists of three widely-separated steerable parabolic dishes and observes at frequencies of both $928 \mathrm{MHz}$ and $1420 \mathrm{MHz}$, and the ESR (single steerable parabolic dish) observes at a frequency of $500 \mathrm{MHz}$. A cross-correlation of signals from simultaneous observations of the same radio source at the same frequency using two different observing sites yields a cross-correlation function (CCF) with a time lag from which solarwind velocities can be obtained (see, e.g., Coles and Harmon, 1978). Cross-correlation of multi-frequency observations from two different sites is also possible (Salpeter, 1967) and have been shown to yield valid results (see, e.g., Fallows et al., 2006; Bisi, 2006). The largest peak in the CCF is caused by the dominant solar-wind stream crossing the line of sight (LOS) ray path from source to observer. Since IPS is sensitive to movement perpendicular to the LOS, the majority of contribution comes from near to the point of closest approach (P-Point or impact parameter) of the LOS ray path to the Sun, but contributions from the tails of the LOS are still significant enough that they cannot be ignored. Since the scattering that gives rise to IPS falls substantially with distance from the Sun (as approximately $R^{-4}$ ), the majority of this contribution comes from near to the point of closest approach (P-Point or impact parameter) of the LOS to the Sun. However, contributions from points further along the line of sight (at greater distances from the Sun) cannot be ignored and a full LOS integration is necessary when modelling the observations. In general, the more density turbulent the material crossing the LOS, the greater the contribution is to the overall signal recorded by these instruments. IPS is sensitive to small-scale density variations on the order of $50-150 \mathrm{~km}$ size at these frequencies; it is these variations which cause the fluctuations in radio signal 
and thus also result in the amplitude scintillation received at Earth. The observing frequencies of the EISCAT UHF antennas allow reliable observations of radio sources with P-Point distances in the approximate range $20 R_{\odot}$ to $100 R_{\odot}$, roughly equivalent to elongations of $5^{\circ}$ to $30^{\circ}$. However, it must be emphasised that these represent the closest points of the lines of sight to the Sun; the full LOS integration will include contributions to the received signal from beyond Earth orbit in the direction of the radio source to the P-Point and from the P-Point to the observer on Earth. The velocities used in this paper are the main CCF peak velocity, i.e. the dominant solar-wind velocity crossing the LOS.

The inner heliosphere can be reconstructed in three dimensions through the use of the University of California, San Diego (UCSD) three-dimensional (3-D) computer assisted tomography (C.A.T.) reconstruction program incorporating a time-dependent kinematic solarwind model (see, e.g., Jackson et al., 2003; Jackson and Hick, 2005; and references therein). This program uses the IPS velocity determinations to reconstruct the 3-D velocity distribution in the inner heliosphere (out to $3 \mathrm{AU}$ ). The 3-D reconstructions in this paper correspond to Carrington rotation (CR) 2055.85 to 2056.85 .

Section 2 gives a brief description of our 3-D time-dependent reconstructions and the model used. Section 3 shows the 3-D reconstruction results and comparison with in-situ velocity measurements from the Solar TErrestrial RElations Observatory (STEREO) (Kaiser, 2005; Kaiser et al., 2008) twin spacecraft (STEREO-A out ahead of the Earth and STEREOB lagging behind the Earth), and the Wind (Ogilvie and Desch, 1997) and Advanced Composition Explorer (ACE) (Stone et al., 1998) spacecraft instrumentation. Section 4 discusses and summarises the results, and provides some conclusions, as well as a look to the future.

\section{3-D Reconstructions}

The 3-D tomographic reconstructions use perspective views of outward-flowing material crossing the IPS observing lines of sight from Earth to the radio source. These IPS data are then fitted iteratively with a kinematic model (conserving mass and mass flux) to refine the fit over 18 iterations to ensure convergence to a final best-fit solution. We then compare the resulting reconstructions with hourly-averaged in-situ measurements from the STEREO Plasma and Suprathermal Ion Composition (STEREO|PLASTIC) (Galvin et al., 2008), the Wind - Solar Wind Experiment (Wind $\mid$ SWE) (Ogilvie et al., 1995), and the ACE - Solar Wind Electron Proton Alpha Monitor (ACE|SWEPAM) (McComas et al., 1998) instrumentation. It should be noted that at this point in the STEREO Mission, the two spacecraft are still relatively close to Earth. Previous comparisons of such reconstructions with other sources of IPS data have been well established (see, e.g., Bisi et al., 2009a and references therein).

The 3-D reconstructions for the EISCAT and ESR data used here have latitude and longitude digital resolution of $20^{\circ} \times 20^{\circ}$, but are smoothed by a Gaussian filter that interpolates temporally and spatially over regions with few data points (see, e.g., Jackson and Hick, 2005). The resolution is ultimately restricted by the number of lines of sight available for the reconstructions. The one-day time cadence has six-hour interpolated increments to yield output four times a day for the modelled structure of the inner heliosphere. The IPS velocity data used as input to the model was limited to observations with determined velocity values of $100 \mathrm{~km} \mathrm{~s}^{-1}$ to $1250 \mathrm{~km} \mathrm{~s}^{-1}$ inclusive, since this gave the best overall broad comparison with in-situ measurements near Earth and revealed good co-rotating structures. The 


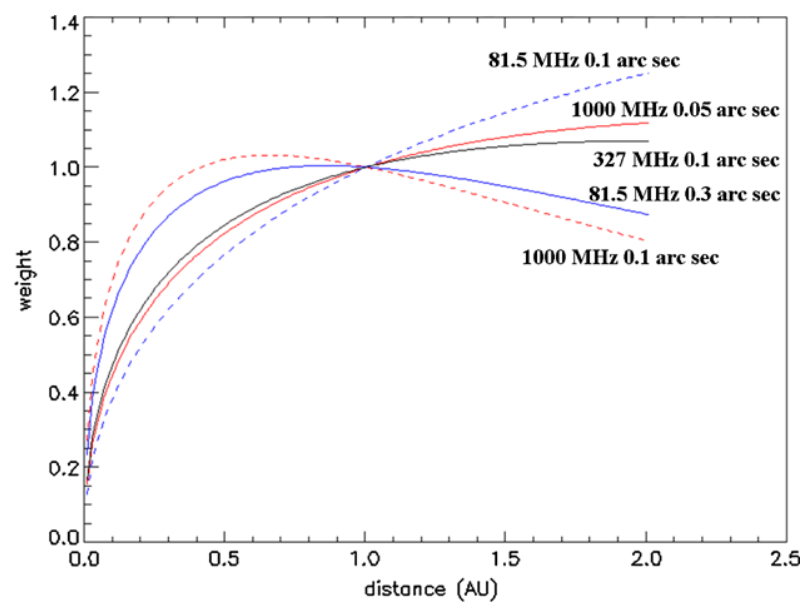

Figure 1 Figure showing the IPS LOS weighting distribution for different observing frequencies with assumed source sizes. The weights are "normalized" to $1 \mathrm{AU}$ and do not include the $R^{-4}$ variation of scattering with distance from the Sun. Thus, this shows the scaling between the Fresnel-scale size and the source diameter. Distance in this plot refers to the distance along the LOS from Earth in the direction to the source. As can be seen, the traditionally-used $327 \mathrm{MHz} 0.1 \mathrm{arc} \mathrm{sec}$ source-size distribution is very similar to the $1000 \mathrm{MHz}$ (approximate average distribution of the three EISCAT/ESR observing frequencies) 0.05 arc sec source size.

IPS LOS weighting distribution for different frequencies and source sizes can be seen in Figure 1. This weighting factor is a result of the IPS response to the Fresnel filtering effect (Coles and Harmon, 1978) and to radio source size, as described in Jackson et al. (1998). The weights are "normalised" to $1 \mathrm{AU}$ and it is the change in value as you go along the LOS in steps that matters, and not the value itself. As can be seen, the approximate average of the EISCAT/ESR observing frequencies (1000 MHz) with the assumed smaller source size $(0.05 \mathrm{arcsec})$ gives a very similar weighting along the LOS as that normally used for $327 \mathrm{MHz}(0.1 \mathrm{arcsec})$ observations. Thus, this has been kept the same for this reconstruction.

\section{Observations and Measurements}

In this paper, we display various time-series plots and images to show the 3-D reconstructed heliosphere in velocity as well as an associated movie showing the inner heliosphere. Figure 2 shows the hourly-averaged in-situ measurements re-averaged to the one-day cadence of the reconstructions compared with extractions from the reconstructed volume at the position of each of the respective spacecraft. Note that at this daily average, there is little difference in velocities measured from one spacecraft to the other (as expected for large-scale velocity structure during this time).

In general, a good comparison exists with all four spacecraft measurements (Wind, ACE, STEREO-A, and STEREO-B), but a few discrepancies also occur. The main discrepancy is seen around 4-8 May 2007 at approximately 1 AU distance from the Sun near the Earth when the four spacecraft are located closely to each other, where an anti-correlation occurs with the in-situ comparisons. This is likely caused by the paucity of observations between 28 April 2007 and 03 May 2007: because EISCAT observations take place close-in to the Sun the impact on the reconstructions at $1 \mathrm{AU}$ are delayed by a full three to four 

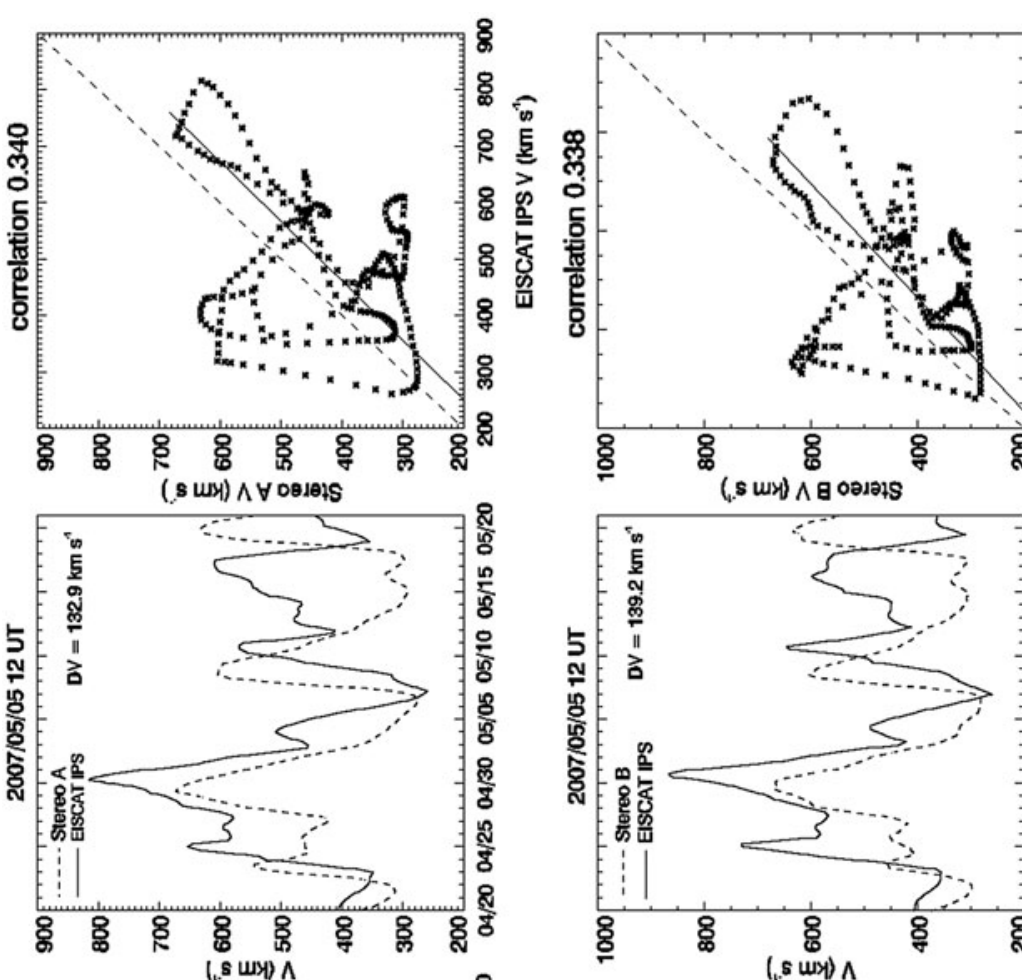

8

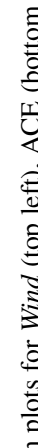

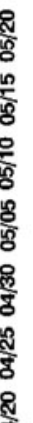

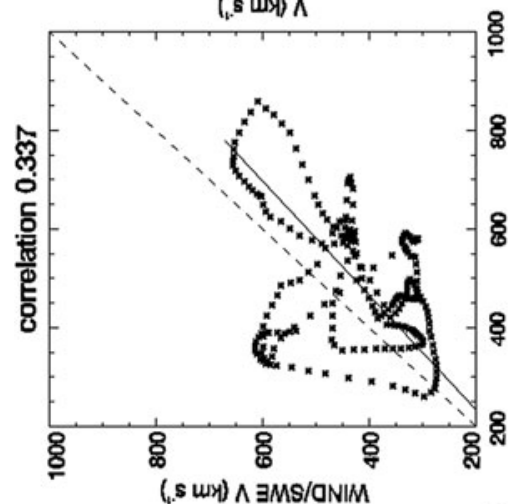

\&

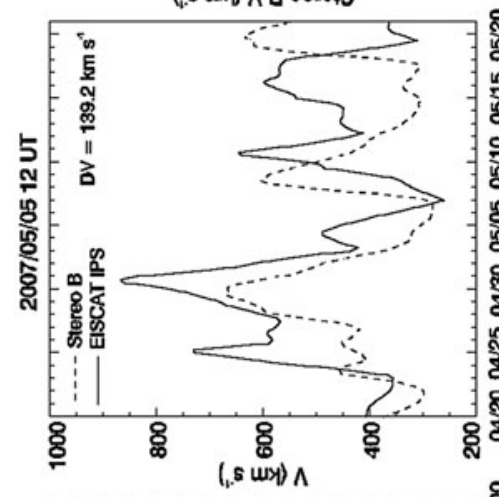

\&ิ क्षे

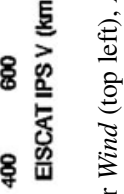

올

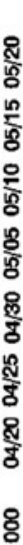

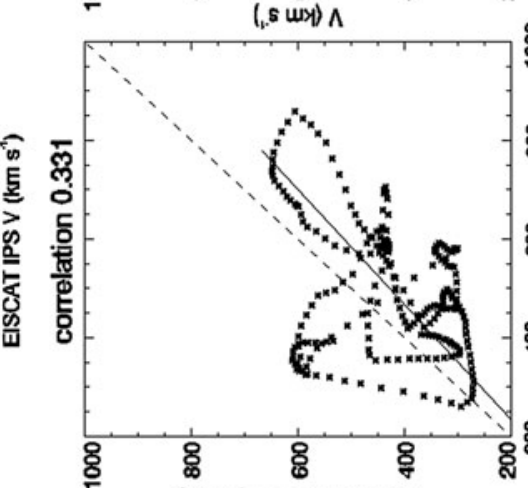

8
8
8

ए

노
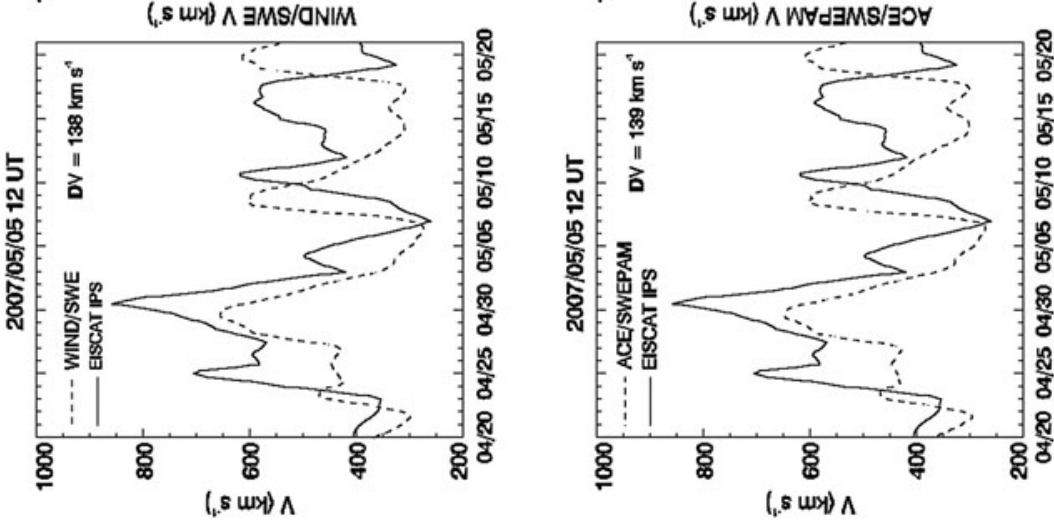

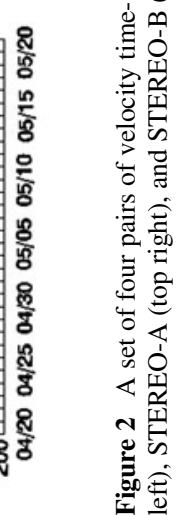




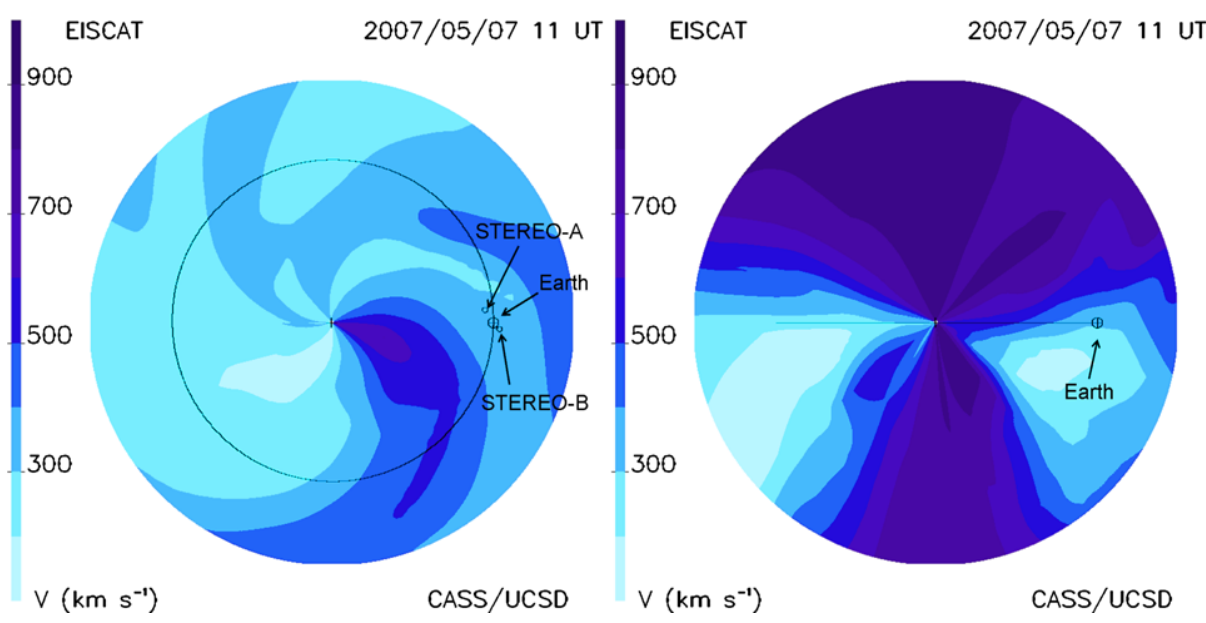

Figure 3 An ecliptic cut (left) showing the velocity structures in the ecliptic at the date and time shown; STEREO-A, Earth and Earth's orbital path, and STEREO-B are also marked in the image at this time. Also shown is a meridional cut (right) where the Earth's position can be seen marked on the right and the line representing the Earth's orbital path superimposed as the side-on view. Note the dipole-looking structure in solar-wind velocity distribution of the meriodional cut with higher velocity over the poles and lower velocity generally in the ecliptic.

days travel time out to $1 \mathrm{AU}$ where we compare our reconstructed values with those measured by the four spacecraft. The tomography ideally requires continuous data to allow consistent reconstruction of the inner heliosphere with high accuracy, and thus to continue to compare well with in-situ measurements (as is shown with good comparison for periods prior to the data gap/shortage where there were a greater number of observations each day).

Figure 3 shows a cut through the 3-D reconstruction in the ecliptic plane (left) and also a cut in the meridional plane (right). The two-dimensional (2-D) view of the ecliptic cut is from directly north out to $1.5 \mathrm{AU}$ at the outer edge, and the 2-D view of the meridional cut is from directly east of the Sun-Earth line and also out to 1.5 AU at the outer edge. The Earth in the ecliptic can be seen to the right of the image along with the two STEREO spacecraft (labelled in the ecliptic-cut image); the Earth's orbit is also shown and marked by the near-circular black line. The Earth can also be seen to the right of the meridional-cut image and its orbit as a horizontal line across the centre.

Figure 4 shows four 3-D views out to around 1.5 AU from the Sun from about $30^{\circ}$ above the ecliptic and $45^{\circ}$ west of the Sun-Earth line, each one day apart starting on 22 April 2007 and ending on 25 April 2007. Clearly shown are the slow-wind portions of the large co-rotating velocity structures and the rotation of these structures spanning this three-day time interval. Of particular interest is the co-rotating stream reproduced, starting near Earth moving to the west of the Sun-Earth line (seen starting in the bottom-left of the top-left image and later nearly at the bottom of the bottom-right image at the end of the threeday period shown). This is of interest because it is seen to rotate a little under $45^{\circ}$ in this three-day length, which is approximately the equivalent amount expected for the $\sim 25$-day Sun-rotation rate in equatorial regions.

The associated movie (in the online version) shows this entire reconstructed period in three dimensions as viewed by a remote observer some $45^{\circ}$ west of the Sun - Earth line and approximately $30^{\circ}$ above the ecliptic plane (as with the Figure 4 images). In this movie, the 

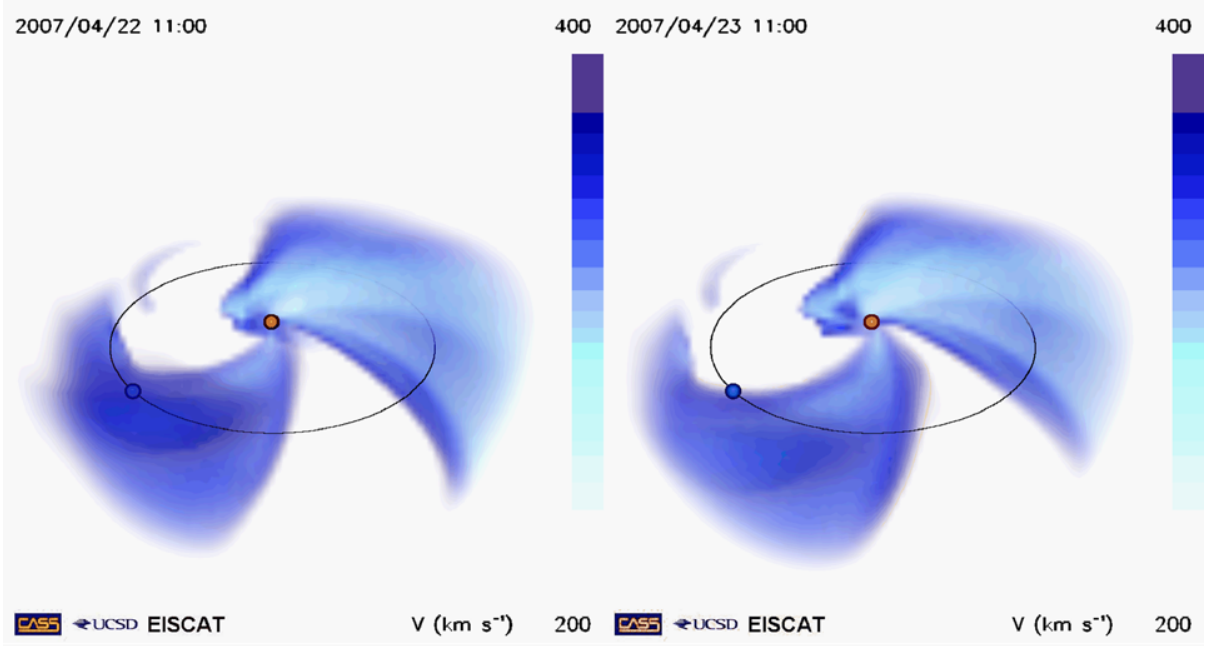

$V\left(\mathrm{~km} \mathrm{~s}^{-1}\right)$
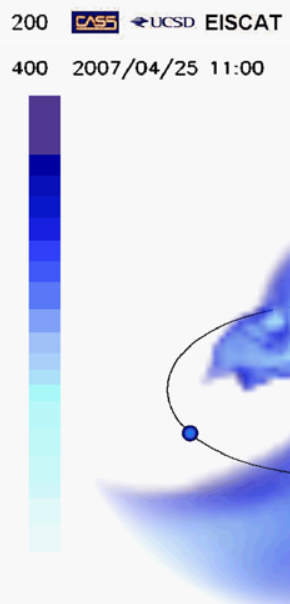

$\mathrm{V}\left(\mathrm{km} \mathrm{s}^{-1}\right)$

200

2007/04/24 11:00

$400 \quad 2007 / 04 / 25 \quad 11: 00$

400
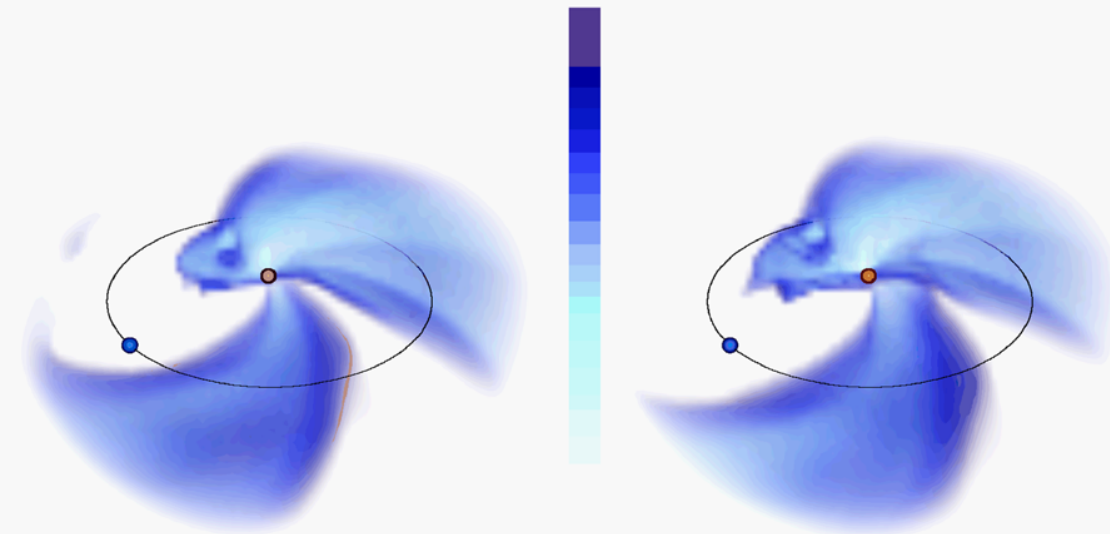

Figure 4 A set of four velocity 3-D views showing the slow-wind portions of co-rotating structures at the dates and times shown (the higher-velocity portions are not shown for clarity of viewing). These images form part of the online movie covering this entire period. The Earth is shown as a blue sphere towards the bottom-left of each image along with its orbital path shown in black. The orange/red sphere in the centre of each image represents the Sun (neither the Earth or the Sun spheres are to scale).

slow-velocity portions of co-rotating structures can be easily seen, as well as a few possibly transient-type features which appear to not co-rotate.

In addition, towards the end of this period, an interplanetary coronal mass ejection (ICME) was detected in the STEREO-A inner Heliospheric Imager (HI-1) which forms part of the STEREO - Sun Earth Connection Coronal and Heliospheric Investigation (SECCHI) instrument suite (Howard et al., 2002, 2008). An example image of this ICME as observed by HI-1A is shown in Figure 5. A clear coronal mass ejection (CME) was also observed by the SOlar and Heliospheric Observatory (SOHO) - Large Angle Spectrometric COronagraph (SOHO|LASCO) (Domingo, Fleck, and Poland, 1995; Brueckner et al., 1995), and by the STEREO - CORoronagraph (COR) instruments on 


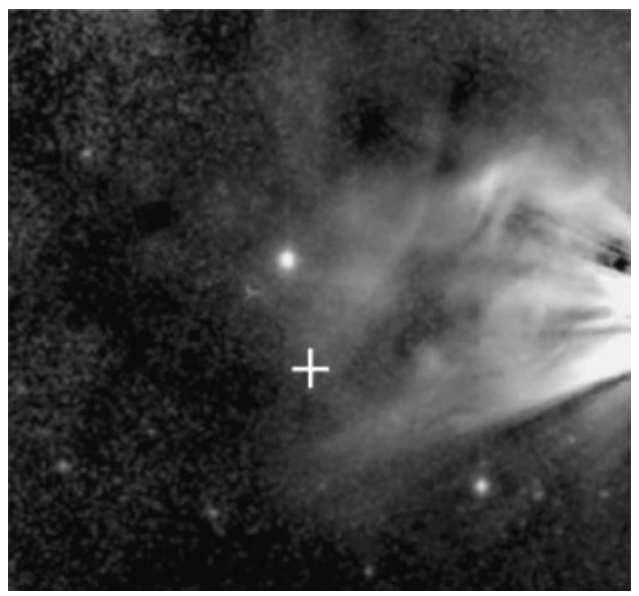

Figure 5 An image of an ICME taken with STEREO HI-1A at the date and time shown covering a 40-minute exposure time. The image is $20^{\circ}$ by $20^{\circ}$ and the Sun is located $\approx 4^{\circ}$ to the right of the image. The white "+" just below the centre of the image marks the projection of the P-Point of and EISCAT observations of IPS using source J0431 + 206 earlier in the same day which showed clear signs of an ICME signature before the front seen in this present image had passed over this projected point. Figure adopted from Dorrian et al. (2009).

Figure 6 An image of the reconstructed velocity relating to the ICME from Figure 5 with the same perspective view and parameters as in Figure 4. The velocity structure that forms a perturbation of the other more-regular patterns is interpreted to be associated with the ICME, and is circled to the left of the Sun.

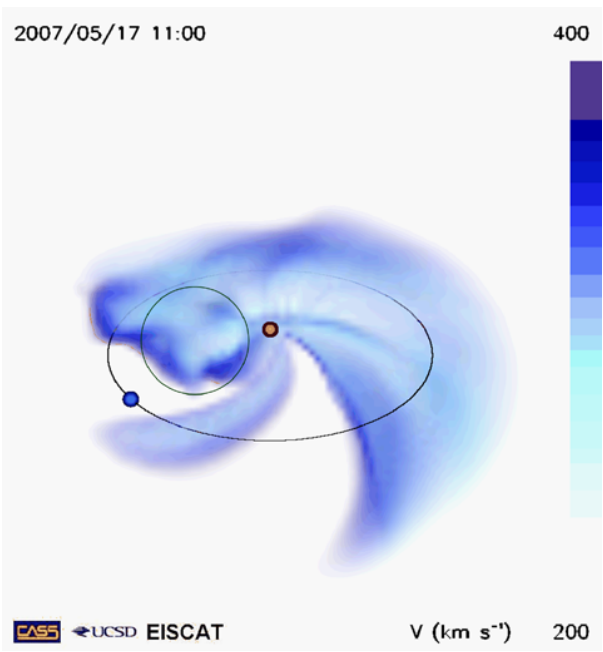

400

STEREO-A (also part of the SECCHI suite). An EISCAT observation of IPS using source J0431 + 206 on 16 May 2007 also shows ICME signatures, but somewhat early for the main ICME front to have intersected the IPS LOS ray path (Dorrian et al., 2009) as seen by STEREO HI-1A.

Looking at the end of this reconstructed period (which ends on 20 May 2007), the ICME can perhaps be seen as a perturbation of the slow solar wind heading between the Earth and east of the Sun as visualised in Figure 6, which has the same perspective view as the images shown in Figure 4 and the associated movie. 


\section{Discussion and Summary}

3-D reconstructions of EISCAT IPS velocity data were preliminarily attempted by Bisi et al. (2007a) using data from 2005. These showed gross-structure comparisons in 2-D and 3-D images compared with another IPS reconstruction of the same period using data from the Solar-Terrestrial Environment Laboratory (STELab) (Kojima and Kakinuma, 1987).

It should be noted that the number of individual EISCAT observations giving reliable velocities were no more than ten on any given day. Also, there were several days with few-to-no suitable observations. The fact that this technique appears to work for so few observations is down to the Gaussian filters: even though the reconstructions have a one-day cadence, the Gaussian filters smooth between places where there are data to a value indicated by a $3-\mathrm{D}$ reconstruction average. This means that detail will inevitably be lost and more structure is likely to exist than is reconstructed. Given this, the 3-D velocity reconstructions using EISCAT IPS velocity data appear surprisingly accurate when compared with "ground-truth" in-situ measurements by near-Earth spacecraft. They display well-defined co-rotating structures, as well as evidence at the end of the observing period of the 16 May 2007 EISCAT and STEREO HI-1A ICME mostly to the east of the Sun - Earth line.

Throughout this time interval of the declining phase of Solar Cycle 23, co-rotating structures were more dominant, and the Sun and inner heliosphere seemed to show signs of similar solar-wind structure akin to that seen in the previous declining phase of Solar Cycle 22 (see, e.g., Breen et al., 1999, 2000). The domination of co-rotating velocity structures is expected for solar-minimum conditions in the declining and minimum phases, as it was between Solar Cycles 22 and 23. It should be noted though, that deeper into the current solarminimum phase, velocity co-rotation became much less dominant and the Sun's dipole-like structure in velocity (Figure 3) became more complex with a "tilted" solar-wind streamer belt (see, e.g., Bisi et al., 2009a, 2009b) and a seeming "lack" of dipole-like structure (Tokumaru et al., 2009).

The ICME in the velocity reconstruction is seen in the 3-D remote-observer view as a perturbation, mostly to the east of the Sun - Earth line, of the spiral-like structure which dominates this interval. In the associated movie, its development can be watched right through to the end of the reconstruction from its onset around 16-17 May 2007. It is similar in its timing from when seen earlier in the day on 16 May 2007 in the STEREO HI-1A images, and is also confirmed as having perhaps an even fainter front from the slightly earlier individual EISCAT observations of source J0431 + 206. Both IPS and STEREO HI observations can be used in a highly-complementary manner to obtain high-time resolution information of transient solar-wind features passing through interplanetary space.

The results displayed in this paper again show the robustness of our model and of the reconstruction technique at UCSD, this time with EISCAT/ESR observations of IPS. They provide a relatively-easy way of viewing the large-scale 3-D structure, at least in velocity in the case of these observations of IPS, of the solar wind throughout a Carrington rotation, as well as when transients occur that result in disturbances through the overall large-scale structure (which in this case was somewhat stable co-rotationally dominated). It would be useful to compare further with the many stream interaction regions detected using EISCAT data around the previous solar minimum by Bisi et al. (2010), and also to look more into the large-scale structure of the fast solar wind, as investigated by Bisi et al. (2007b), with perhaps STELab IPS reconstructions since EISCAT data at those times are not likely numerous enough to form a successful reconstruction.

Looking to the future however, attempts to enable an even more focussed campaign with EISCAT/ESR observations of IPS (if the sources are available) would be worthwhile to test 
out its success again; particularly now that the initial and expected rising phase of Solar Cycle 24 has commenced, and transients should become more prevalent. The modification of taking into account the small differences in distribution weighting along the LOS for each observation at the different frequencies used by EISCAT and the ESR, and the assumed source sizes for each would also be worthwhile in light of the aims of using this 3-D reconstruction technique for future multi-frequency IPS data. These future lower-frequency observations will be taken by the soon-to-be-operational LOw Frequency ARray (LOFAR) in Europe (with its core in The Netherlands) and also the United States (US) National Science Foundation (NSF) funded Murchison Widefield Array (MWA) in western Australia (see, e.g., Salah et al., 2005; Oberoi and Benkevitch, 2010, in this issue).

Acknowledgements The University of California, San Diego (UCSD) authors acknowledge the United States (US) National Science Foundation (NSF) (grants ATM0852246 and ATM0925023) and the US Air Force Office of Scientific Research (AFOSR) (grant FA9550-06-1-0107) for their funding while working on these analyses. The Aberystwyth University and Queen's University Belfast authors acknowledge United Kingdom (UK) funding from the Science and Technologies Facilities Council (STFC). The authors would like to thank the directors and staff of EISCAT/ESR for their help and for the EISCAT/ESR data used in this study. EISCAT is supported by the scientific research councils of China, Finland, France, Germany, Japan, Norway, Sweden, and the United Kingdom. We would also like to extend particular thanks to W.A. Coles and B.J. Rickett of UCSD, for making available their analysis routines, expertise, and advice to us when analysing the EISCAT and ESR IPS data. The authors also thank and acknowledge the STEREO, Wind, and ACE instrument groups for making their data freely available for scientific use. The STEREO|SECCHI data used here are produced by an international consortium of the Naval Research Laboratory (USA), Lockheed Martin Solar and Astrophysics Lab (USA), NASA Goddard Space Flight Center (USA), Rutherford Appleton Laboratory (UK), University of Birmingham (UK), Max-Planck-Institut für Sonnensystemforschung (Germany), Centre Spatiale de Liège (Belgium), Institut d'Optique Théorique et Appliquée (France), Institut d'Astrophysique Spatiale (France).

Open Access This article is distributed under the terms of the Creative Commons Attribution Noncommercial License which permits any noncommercial use, distribution, and reproduction in any medium, provided the original author(s) and source are credited.

\section{References}

Bisi, M.M.: 2006, Interplanetary scintillation studies of the large-scale structure of the solar wind, PhD Thesis, The University of Wales, Aberystwyth.

Bisi, M.M., Jackson, B.V., Fallows, R.A., Breen, A.R., Hick, P.P., Wannberg, G., Thomasson, P., Jordan, C.A., Dorrian, G.D.: 2007a, Combined STELab, EISCAT, ESR, and MERLIN IPS observations of the solar wind. Proc. SPIE 6689, 668911-1. doi:10.1117/12.735443.

Bisi, M.M., Fallows, R.A., Breen, A.R., Habbal, S.R., Jones, R.A.: 2007b, Large-scale structure of the fast solar wind. J. Geophys. Res. 112, A06101. doi:10.1029/2006JA012166.

Bisi, M.M., Jackson, B.V., Buffington, A., Clover, J.M., Hick, P.P., Tokumaru, M.: 2009a, Low-Resolution STELab IPS 3D reconstructions of the whole heliosphere interval and comparison with in-ecliptic solar wind measurements from STEREO and Wind instrumentation. Solar Phys. 256, 201-217. doi:10.1007/s11207-009-9350-9.

Bisi, M.M., Jackson, B.V., Clover, J.M., Hick, P.P., Buffington, A., Tokumaru, M.: 2009b, A summary of 3-D reconstructions of the whole heliosphere interval and comparison with in-ecliptic solar wind measurements from STEREO, ACE, and Wind instrumentation. In: Corbett, I. F. (ed.) Highlights of Astronomy, XXVIIth IAU General Assembly 15, 119.

Bisi, M.M., Fallows, R.A., Breen, A.R., O’Neill, I.J.: 2010, Interplanetary scintillation observations of stream interaction regions in the solar wind. Solar Phys. 261, 149-172. doi:10.1007/s11207-009-9471-1.

Breen, A.R., Mikić, Z., Linker, J.A., Lazarus, A.J., Thompson, B.J., Biesecker, D.A., Moran, P.J., Varley, C.A., Williams, P.J.S., Lecinski, A.: 1999, Interplanetary scintillation measurements of the solar wind during whole Sun month: comparisons with coronal and in situ observations. J. Geophys. Res. 104, $9847-9870$. 
Breen, A.R., Thompson, B.J., Kojima, M., Biesecker, D.A., Canals, A., Fallows, R.A., Linker, J.A., Lazarus, A.J., Lecinski, A., Mikić, Z., Moran, P.J., Williams, P.J.S.: 2000, Measurements of the solar wind over a wide range of heliocentric distances - a comparison of results from the first three whole Sun months. J. Atmos. Terr. Phys. 62, $1527-1543$.

Brueckner, G.E., Howard, R.A., Koomen, M.J., Korendyke, C.M., Michels, D.J., Moses, J.D., Socker, D.G., Dere, K.P., Lamy, P.L., Llebaria, A., Bout, M.V., Schwenn, R., Simnett, G.M., Bedford, D.K., Eyles, C.J.: 1995, The large angle spectroscopic coronagraph (LASCO). Solar Phys. 162, 357-402.

Coles, W.A., Harmon, J.K.: 1978, Interplanetary scintillation measurements of the electron density power spectrum in the solar wind. J. Geophys. Res. 83(A4), 1413-1420.

Domingo, V., Fleck, B., Poland, A.I.: 1995, SOHO: the solar and heliospheric observatory. Space Sci. Rev. 72, $81-84$.

Dorrian, G.D., Breen, A.R., Davies, J.A., Rouillard, A.P., Bisi, M.M., Whittaker, I., Fallows, R.A.: 2009, Simultaneous heliospheric imager and interplanetary scintillation observations of CMEs and CIRs, STEREO SWG 19, Pasadena, CA, USA, oral presentation by M.M. Bisi.

Fallows, R.A., Breen, A.R., Bisi, M.M., Jones, R.A., Wannberg, G.: 2006, Dual-frequency interplanetary scintillation observations of the solar wind. Geophys. Res. Lett. 33, 11106. doi:10.1029/2006GL025804.

Galvin, A.B., Kistler, L.M., Popecki, M.A., Farrugia, C.J., Simunac, K.D.C., Ellis, L., Möbius, E., Lee, M.A., Boehm, M., Carroll, J., Crawshaw, A., Conti, M., Demaine, P., Ellis, S., Gaidos, J.A., Googins, J., Granoff, M., Gustafson, A., Heirtzler, D., King, B., Knauss, U., Levasseur, J., Longworth, S., Singer, K., Turco, S., Vachon, P., Vosbury, M., Widholm, M., Blush, L.M., Karrer, R., Bochsler, P., Daoudi, H., Etter, A., Fischer, J., Jost, J., Opitz, A., Sigrist, M., Wurz, P., Klecker, B., Ertl, M., Seidenschwang, E., Wimmer-Schweingruber, R.F., Koeten, M., Thompson, B., Steinfeld, D.: 2008, The plasma and suprathermal ion composition (PLASTIC) investigation on the STEREO observatories. Space Sci. Rev. 136, 437 - 486. doi:10.1007/s11214-007-9296-x.

Howard, R.A., Moses, J.D., Socker, D.G., Dere, K.P., Cook, J.W.: 2002, Sun Earth connection coronal and heliospheric investigation (SECCHI). Space Sci. Rev. 29, 2017 - 2026.

Howard, R.A., Moses, J.D., Vourlidas, A., Newmark, J.S., Socker, D.G., Plunkett, S.P., Korendyke, C.M., Cook, J.W., Hurley, A., Davila, J.M., Thompson, W.T., St. Cyr, O.C., Mentzell, E., Mehalick, K., Lemen, J.R., Wuelser, J.P., Duncan, D.W., Tarbell, T.D., Wolfson, C.J., Moore, A., Harrison, R.A., Waltham, N.R., Lang, J., Davis, C.J., Eyles, C.J., Mapson-Menard, H., Simnett, G.M., Halain, J.P., Defise, J.M., Mazy, E., Rochus, P., Mercier, R., Ravet, M.F., Delmotte, F., Auchere, F., Delaboudinière, J.P., Bothmer, V., Deutsch, W., Wang, D., Rich, N., Cooper, S., Stephens, V., Maahs, G., Baugh, R., McMullin, D., Carter, T.: 2008, Sun Earth connection coronal and heliospheric investigation (SECCHI). Space Sci. Rev. 136, $67-115$. doi:10.1007/s11214-008-9341-4.

Jackson, B.V., Hick, P.P.: 2005, Three-dimensional tomography of interplanetary disturbances. In: Gary, D., Keller, C.U. (eds.) Solar and Space Weather Radiophysics: Current Status and Future Developments, Astrophys. and Space Sci. Lib. 314, Kluwer Academic, Dordrecht, 355 - 386, Chap. 17.

Jackson, B.V., Hick, P.P., Kojima, M., Yokobe, A.: 1998, Heliospheric tomography using interplanetary scintillation observations, 1. Combined Nagoya and Cambridge data. J. Geophys. Res. 103(A6), 1204912067.

Jackson, B.V., Hick, P.P., Buffington, A., Kojima, M., Tokumaru, M., Fujiki, K., Ohmi, T., Yamashita, M.: 2003, Time-dependent tomography of hemispheric features using interplanetary scintillation (IPS) remote-sensing observations. In: Velli, M., Bruno, R., Malara, F., Bucci, B. (eds.) Solar Wind Ten, American Institute of Physics Conference Series 679, ATP, New York, 75 - 78. doi:10.1063/1.1618545.

Kaiser, M.L.: 2005, The STEREO mission: an overview. Space Sci. Rev. 36, 1483 - 1488. doi:10.1016/j.asr. 2004.12.066.

Kaiser, M.L., Kucera, T.A., Davila, J.M., St. Cyr, O.C., Guhathakurta, M., Christian, E.: 2008, The STEREO mission: an introduction. Space Sci. Rev. 136, 5 - 16. doi:10.1007/s11214-007-9277-0.

Kojima, M., Kakinuma, T.: 1987, Solar cycle evolution of solar wind speed structure between 1973 and 1985 observed with the interplanetary scintillation method. J. Geophys. Res. 92, $7269-7279$.

McComas, D.J., Bame, S.J., Barker, P., Feldman, W.C., Phillips, J.L., Riley, P., Griffee, J.W.: 1998, Solar wind electron proton alpha monitor (SWEPAM) for the advanced composition explorer. Space Sci. Rev. 86, 563 - 612. doi:10.1023/A:1005040232597.

Oberoi, D., Benkevitch, L.: 2010, Remote sensing of the heliosphere with the Murchison widefield array. Solar Phys. doi:10.1007/s11207-010-9580-x.

Ogilvie, K.W., Desch, M.D.: 1997, The wind spacecraft and its early scientific results. Space Sci. Rev. 20, $559-568$.

Ogilvie, K.W., Chornay, D.J., Fritzenreiter, R.J., Hunsaker, F., Keller, J., Lobell, J., Miller, G., Scudder, J.D., Sittler, E.C. Jr., Torbert, R.B., Bodet, D., Needell, G., Lazarus, A.J., Steinberg, J.T., Tappan, J.H., Mavretic, A., Gergin, E.: 1995, SWE, a comprehensive plasma instrument for the wind spacecraft. Space Sci. Rev. 71, 55-77. 
Rishbeth, H., Williams, P.J.S.: 1985, Ionospheric Radar: the system and its early results. Mon. Not. Roy. Astron. Soc. 26, $478-512$.

Salah, J.E., Lonsdale, C.J., Oberoi, D., Cappallo, R.J., Kasper, J.C.: 2005, Space weather capabilities of low frequency radio arrays. In: Fineschi, S., Viereck, R.A. (eds.) Society of Photo-Optical Instrumentation Engineers (SPIE) Conference Series, Presented at the Society of Photo-Optical Instrumentation Engineers (SPIE) Conference 5901, 124 - 134. doi:10.1117/12.613448.

Salpeter, E.E.: 1967, Interplanetary scintillations. I. Theory. Astrophys. J. 147, 433.

Stone, E.C., Frandsen, A.M., Mewaldt, R.A., Christian, E.R., Margolies, D., Ormes, J.F., Snow, F.: 1998, The advanced composition explorer. Space Sci. Rev. 86, 1 - 22. doi:10.1023/A:1005082526237.

Tokumaru, M., Kojima, M., Fujiki, K., Hayashi, K.: 2009, Non-dipolar solar wind structure observed in the cycle 23/24 minimum. Geophys. Res. Lett. 36, L091001.

Wannberg, G., Wolf, I., Vanhainen, L.G., Koskenniemi, K., Röttger, J., Postila, M., Markkanen, J., Jacobsen, R., Stenberg, A., Larsen, R., Eliassen, S., Heck, S., Huuskonen, A.: 1997, The EISCAT Svalbard radar, a case study in modern incoherent scatter radar system design. Radio Sci. 32, 2283-2307.

Wannberg, G., Vanhainen, L.G., Westman, A., Breen, A.R., Williams, P.J.S.: 2002, The new $1420 \mathrm{MHz}$ dual polarisation interplanetary scintillation (IPS) facility at EISCAT. In: Conference Proceedings, Union of Radio Scientists (URSI). 